\title{
Healthcare Resource Utilization Following a Discharge Against Medical Advice: An Analysis of Commercially Insured Adults
}

\author{
Aakash Bipin Gandhi, BPharm¹, Eberechukwu Onukwugha, PhD, MS, Jacquelyn McRae, PharmD¹, David Alfandre, MD²
}

'University of Maryland School of Pharmacy, Baltimore, Maryland; ${ }^{2}$ A N National Center for Ethics in Health Care, NYU School of Medicine, New York, New York.

BACKGROUND: A discharge against medical advice (DAMA) is associated with adverse health outcomes. Its association with postdischarge healthcare resource utilization (HcRU) outside an inpatient setting is unknown. This information can help us understand how a DAMA may affect healthcare-seeking behavior following a hospital stay. We evaluated the relationship between a DAMA and 30-day postdischarge HcRU.

METHODS: This retrospective cohort study uses a $10 \%$ random sample of enrollees in the IQVIA PharMetrics ${ }^{\circledR}$ Plus database. We included individuals aged 18 to 64 years with an inpatient admission during 2007-2015 and continuous insurance coverage. We defined comparison groups as DAMA and routine discharge. Both groups were matched on baseline covariates. We quantified the association between a DAMA and 30-day HcRU, as well as 90-day for sensitivity analysis, with use of generalized linear models for binary outcomes (inpatient readmissions, emergency department [ED] visits) and count outcomes (physician office visits, nonphysician outpatient encounters, prescription drug fills).

RESULTS: Of the 457,530 individuals in the unmatched sample, 2,245 (0.5\%) had a DAMA. In the matched sample, a DAMA was positively associated with an ED visit (adjusted odds ratio, 2.28; $95 \%$ confidence interval, 1.90-2.72) but not with an inpatient readmission. There were no differences between groups based on the count outcomes. A DAMA was positively associated with 90-day HcRU (ie, inpatient readmission, ED visit, and prescription drug fills).

CONCLUSION: The relationship between a DAMA and $\mathrm{HcRU}$ varied with the HcRU category and postdischarge time interval. This examination of HcRU in the inpatient and outpatient settings provides important information about outcomes following a DAMA. Journal of Hospital Medicine 2020;15:716-722. (C) 2020 Society of Hospital Medicine

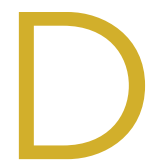

ischarges against medical advice (DAMAs), in which a patient leaves the hospital prior to a physician-recommended endpoint, represent approximately $1 \%$ to $2 \%$ of inpatient discharges in the United States. ${ }^{1}$ When compared with routine discharges, a DAMA is associated with adverse clinical consequences, including an increased risk of all-cause mortality. ${ }^{2,3}$ Additionally, due to incomplete care, a DAMA may result in increased healthcare resource utilization ( $\mathrm{HcRU})$, including the use of inpatient, emergency department (ED), and outpatient services in the postdischarge period. Quantifying these relationships can provide important information regarding an individual's healthcare-seeking behavior following a DAMA.

Prior literature has focused on the association between a DAMA and the risk of inpatient readmission. Relative to routine discharges, a DAMA is associated with a 1.5 to 2 times increased risk of a 30-day readmission. ${ }^{3-9}$ However, these estimates are based on mixed-payer populations primarily com-

*Corresponding Author: Eberechukwu Onukwugha, PhD, MS; Email: eonukwug@rx.umaryland.edu; Telephone: 410-706-8981.

Published online first November 18, 2020.

Find additional supporting information in the online version of this article.

Received: April 22, 2020; Revised: August 3, 2020; Accepted: August 4, 2020

๑ 2020 Society of Hospital Medicine DOI 10.12788/jhm.3516 posed (65\%-80\%) of individuals with public (Medicaid, Medicare) or no insurance. Further, they do not differentiate this association by payer type. It is unclear if prior results apply to commercially insured adults. These individuals represent a small but nonnegligible proportion (19\%) of all DAMAs in the United States. ${ }^{10}$ Quantifying relationships among commercially insured adults can help advance our understanding of readmission patterns in the DAMA population.

There is limited evidence regarding the relationship between a DAMA and outpatient HcRU in the postdischarge period. Use of ED services after a DAMA has been explored only in specific disease populations such as asthma. ${ }^{4}$ Additionally, prior studies have reported a reduced frequency in the receipt of medication prescriptions and outpatient follow-up plans among individuals with a DAMA at the time of discharge..$^{11,12}$ Whether these practices translate to altered patterns of postdischarge prescription drug fills or use of outpatient services is not known.

To address these substantive gaps in the literature, the present study evaluates the association between a DAMA and allcause HcRU in the postdischarge period among commercially insured adults. We examined HcRU across all points of service including inpatient readmissions, ED visits, physician office visits, nonphysician outpatient encounters, and prescription drug fills. These results can serve as a benchmark for comparison to future studies on DAMAs among publicly insured or uninsured 
individuals. Furthermore, such knowledge can help providers, payers, and policy planners make evidence-based decisions regarding postdischarge healthcare delivery.

\section{METHODS}

\section{Data Source}

This retrospective study used a 10\% random sample of enrollees in the IQVIA PharMetrics ${ }^{\circledR}$ Plus database (purchased by University of Maryland, Baltimore, under license from IOVIA). The database is composed of fully adjudicated claims and enrollment information from over 70 contributing US health plans and self-insured employer groups for over 140 million unique enrollees from 2006 onward. The enrollee population is generally representative of the commercially insured population that is younger than 65 years of age (with a subset of commercial Medicare and Medicaid) with respect to age and gender.

The database allows longitudinal follow-up for individuals using three files: medical claims, pharmacy claims, and insurance eligibility. The average length of enrollment is 39 months. The claims data represent payments to providers for services rendered to individuals covered by health plans. The medical claims file contains information on diagnostic and therapeutic services rendered in the inpatient and outpatient settings. The pharmacy claims file captures data on prescription drugs dispensed in retail and mail-order settings. The eligibility file contains demographic and insurance eligibility information for individuals.

\section{Study Population}

We identified all individuals aged 18 to 64 years with an inpatient admission record between January 1, 2007, and December 31,2015 . All individuals with continuous medical and prescription drug coverage from 6 months prior to the hospital admission date (baseline period) through 30 days following the discharge date (follow-up period) were included. Inpatient admissions with a missing discharge disposition or those that resulted in in-hospital death, discharge to a short-term hospital, skilled nursing facility, intermediate care facility, or any other type of facility were not considered for analysis. Only the first eligible inpatient admission was considered for analysis.

\section{Main Predictor Variable}

Individuals with a DAMA were analyzed as the case group. A DAMA was identified using the "Patient Status Code" variable, which represents the discharge disposition of each individual. Individuals who were discharged to home/self-care or discharged to a home health organization formed the control group (hereafter referred to as routine discharge).

\section{Demographic, Clinical, and Hospitalization Characteristics}

An individual's age, sex, and region of residence were determined at the date of hospital admission. The Elixhauser algorithm was used to categorize comorbid conditions (as scores of $0,1-2, \geq 3$ depending on number of comorbidities) based on International Classification of Diseases, Ninth Revision, Clinical Modification, diagnosis codes during the baseline period. ${ }^{13,14}$
The following characteristics of each individual's eligible inpatient admission were captured: year, timing (weekday or weekend), length of stay (LOS, measured in days), and receipt of a surgical procedure.

\section{Outcomes}

All-cause HcRU was identified during the 30-day postdischarge period. Specifically, we identified inpatient readmissions, ED visits, physician office visits, nonphysician outpatient encounters (for example, pathology, radiology, outpatient surgical services), and prescription drug fills. Binary variables (yes or no) were created for inpatient readmissions and ED visits while the remaining $\mathrm{HcRU}$ categories (ie, physician office visits, nonphysician outpatient encounters, and prescription drug fills) were analyzed as count variables. In the sensitivity analyses, we provide results for $\mathrm{HcRU}$ outcomes among a subgroup of individuals who had at least 90 days of continuous medical and prescription drug benefits following the hospital discharge.

\section{Statistical Analysis \\ Descriptive Analysis}

Measures of interest were reported using summary statistics depending on the nature of the variable. Continuous variables were described using $t$ tests, and categorical variables were described using chi-square tests.

\section{Propensity Score Matching}

Cases and controls were matched using a 1:1 greedy matching algorithm based on propensity scores. ${ }^{15}$ We developed propensity scores based on confounders that we hypothesized would be associated with a DAMA and postdischarge HcRU. The propensity score model included the following variables: age, sex, region of residence, Elixhauser comorbidity index score, year of admission, timing of admission, LOS, and presence of any surgical procedure during the inpatient admission. The best match between cases and controls was determined based on the absolute difference in their propensity scores, which allowed for a maximal caliper width of 0.2 of the standard deviation of the logit of the propensity score. ${ }^{16}$ A standardized difference value of less than 0.1 was used to assess balance in baseline patient and hospital characteristics between cases and controls consistent with prior literature. ${ }^{17,18}$ Proportions and balance, as measured by standardized differences between baseline covariates across cases and controls in the matched sample, are displayed in tabular format (Appendix Table 1).

\section{Healthcare Resource Utilization}

We estimated the adjusted odds ratio (AOR) using a logistic regression model. The AOR quantified the association between a DAMA and the prevalence of all-cause inpatient readmissions and ED visits during the 30-day postdischarge period. We estimated incident rate ratios (IRR) for count outcomes. Given the large number of individuals with no physician office visits, nonphysician outpatient encounters, or prescription drug fills, we estimated model parameters for IRRs using a 
TABLE 1. Demographic, Clinical, and Hospitalization Characteristics of the Unmatched Sample

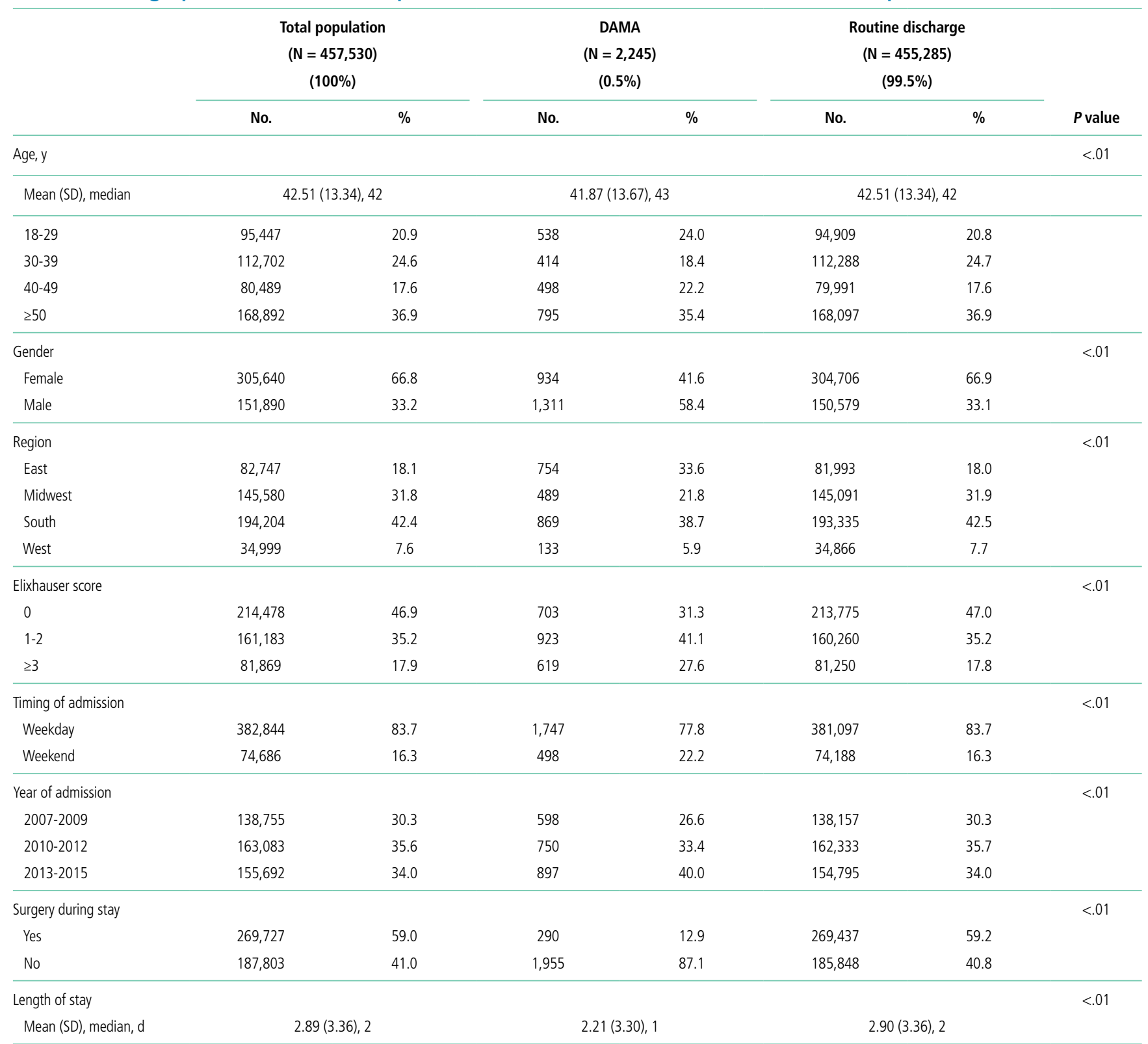

aSome percentages do not sum to $100 \%$ due to rounding.

Abbreviations: d, day; DAMA, discharge against medical advice; SD, standard deviation.

finite mixture negative binomial hurdle model. ${ }^{19}$ We considered the data to represent a mixture of a constant distribution (which always generates zero counts) and a zero-truncated distribution (which always generates nonzero counts). The finite mixture count models include two outcomes: the mixing probabilities and the count distribution. The mixing probabilities quantify the probability that an observation for the HcRU category will be drawn from either the constant distribution (with mass at zero) or the count distribution. Conditional on having positive values, a zero-truncated generalized linear model (GLM) governs the count variable. Compared with other GLM specifications (eg, Poisson, negative binomial, zero-inflated), the negative binomial hurdle model presented the best-fitting model across several information criteria statistics (Appendix Figures 1-3 and Appendix Tables 2-4).

The GLM results provided IRR for the counts of HcRU. Ratios were interpreted as evidence of increased $\mathrm{HcRU}(\mathrm{IRR} \geq 1.0)$ or decreased HcRU (IRR < 1.0) among individuals with a DAMA compared with those discharged routinely. For all HcRU analyses, we reported results for the matched sample. All analyses were conducted using SAS version 9.4 (SAS Institute), and statistical significance was determined at $\alpha=.05$. The study received the University of Maryland, Baltimore, Institutional Review Board approval (HP-00081497). 
TABLE 2. Summary Statistics for HcRU During the 30-day Postdischarge Perioda

\begin{tabular}{|c|c|c|c|c|c|c|}
\hline & \multicolumn{2}{|c|}{$\begin{array}{l}\text { Total population } \\
\quad(\mathrm{N}=4,490)\end{array}$} & \multicolumn{2}{|c|}{$\begin{array}{c}\text { DAMA } \\
(\mathrm{N}=2,245)\end{array}$} & \multicolumn{2}{|c|}{$\begin{array}{l}\text { Routine discharge } \\
\quad(\mathrm{N}=2,245)\end{array}$} \\
\hline & Mean (SD) & Median & Mean (SD) & Median & Mean (SD) & Median \\
\hline Inpatient readmissions & $0.24(0.54)$ & 0 & $0.24(0.55)$ & 0 & $0.21(0.46)$ & 0 \\
\hline Emergency department visits & $0.20(0.60)$ & 0 & $0.28(0.71)$ & 0 & $0.12(0.45)$ & 0 \\
\hline Nonphysician outpatient encounters & $2.43(3.46)$ & 1 & $2.28(3.32)$ & 1 & $2.59(3.60)$ & 1 \\
\hline Prescription drug fills & $3.78(4.04)$ & 3 & $3.57(4.18)$ & 2 & $3.99(3.87)$ & 3 \\
\hline
\end{tabular}

${ }^{a}$ A sample of patients with a routine discharge were matched with those who had a DAMA for this comparison.

Abbreviations: HcRU, healthcare resource utilization; DAMA, discharge against medical advice; SD, standard deviation.

TABLE 3. Adjusted Odds Ratios for Binary Outcomes During 30-Day Postdischarge Perioda

\begin{tabular}{lcccc}
\hline & AOR & $95 \% \mathrm{Cl}$ & P value \\
\hline Inpatient readmissions & 1.06 & $0.91-1.23$ & .47 \\
\hline Emergency department visits & 2.28 & $1.90-2.72$ & $<.01$ \\
\hline
\end{tabular}

a"Binary outcomes" were yes/no outcomes for a population discharged against medical advice versus a matched sample discharged routinely. Abbreviation: $A O R$, adjusted odds ratio.

TABLE 4. Adjusted IRR for Count Outcomes During 30-Day Postdischarge Perioda

\begin{tabular}{llll}
\hline & IRR & $95 \% \mathrm{Cl}$ & $\boldsymbol{P}$ value \\
\hline Physician office visits & 1.01 & $0.91-1.11$ & .88 \\
\hline Nonphysician outpatient encounters & 0.89 & $0.78-1.00$ & .05 \\
\hline Prescription drug fills & 1.03 & $0.97-1.09$ & .35 \\
\hline
\end{tabular}

${ }^{2}$ This was a comparison of count outcomes for a population discharged against medical advice versus a matched sample discharged routinely. Abbreviation: IRR, incident rate ratio.

\section{RESULTS}

The unmatched sample included 457,530 individuals, of whom $0.5 \%$ had a DAMA. A consort diagram illustrating cohort inclusion and exclusion criteria is presented in Appendix Figure 4. Demographic, clinical, and inpatient admission characteristics of the unmatched sample and for subgroups defined by discharge status are displayed in Table 1. In the unmatched sample, the median age at admission was higher for individuals with a DAMA than it was for those discharged routinely (43 vs 42 years, respectively), and the proportion of males was higher among those with a DAMA $(58.4 \%$ vs $33.1 \%)$. There were statistically significant differences based on the geographic region of residence and the comorbidity burden across both groups. The median LOS was shorter ( 1 day vs 2 days), the proportion of weekend admissions was higher (22.2\% vs $16.3 \%$ ), and the proportion of inpatient surgical procedures was lower $(12.9 \%$ vs $59.2 \%$ ) among those with a DAMA compared with that among those with routine discharges. The propensity score-matched sample included 2,245 cases and 2,245 controls (Appendix Table 1). Standardized differences for all baseline factors were less than 0.1 , indicating that cases and controls were matched on the included baseline factors.

\section{Summary Statistics: Proportions and Counts}

Across the DAMA and routine discharge groups, the proportion of individuals with a 30-day inpatient readmission was similar (19.5\% vs $18.7 \% ; P=.47)$, whereas the proportion with an ED visit was higher $(18.6 \%$ vs $9.1 \% ; P<.01)$. There were no differences in the median number of inpatient readmissions (median, 0) and ED visits (median, 0) across both groups. Individuals with a DAMA and those discharged routinely displayed similar median counts of 30-day physician office (median, 1) and nonphysician outpatient encounters (median, 1) (Table 2). Individuals with a DAMA displayed a lower median number of 
prescription drug fills (median, 2 vs 3 ) than that among those with a routine discharge (Table 2 ).

\section{Main Analysis: Thirty-Day Healthcare Resource Utilization}

The associations between a DAMA and 30-day inpatient readmissions and ED visits based on the matched sample are presented in Table 3. Individuals with a DAMA had increased odds for an ED visit (AOR, 2.28; 95\% Cl, 1.90-2.72) but no significant difference in the odds of a 30-day inpatient readmission (AOR, 1.06; 95\% Cl, 0.91-1.23) compared with those discharged routinely.

The association between a DAMA and count HcRU outcomes is presented in Table 4. Compared with those discharged routinely, individuals with a DAMA displayed no significant difference in rates for physician office visits (IRR, 1.01; $95 \% \mathrm{Cl}, 0.91-1.11)$, nonphysician outpatient encounters (IRR, $0.89 ; 95 \% \mathrm{Cl}, 0.78-1.00)$, and prescription drug fills (IRR, 1.03; $95 \% \mathrm{Cl}, 0.97-1.09)$ during the 30-day postdischarge period.

\section{Sensitivity Analysis: Ninety-Day Healthcare Resource Utilization}

Relative to those discharged routinely, individuals with a DAMA had statistically significant increased odds of 90-day inpatient readmissions ( $A O R, 1.18 ; 95 \% \mathrm{Cl}, 1.02-1.36)$, odds of $E D$ visits $(A O R, 2.16 ; 95 \% \mathrm{Cl}, 1.85-2.51)$, and rates of prescription drug fills (IRR, 1.32; 95\% Cl, 1.29-1.35). No statistically significant differences were observed in the rates of physician office visits and nonphysician outpatient encounters across both groups.

\section{DISCUSSION}

In this commercially insured sample of working age individuals, we identified an association between a DAMA and the likelihood and intensity of postdischarge HcRU. The direction of the association varied across categories of $\mathrm{HcRU}$ and the duration of follow-up. A DAMA was associated with increased odds of 30-day ED visits but not 30-day readmissions compared with routine discharges. No significant differences were observed in the rates of 30-day physician office visits, nonphysician outpatient encounters, and prescription drug fills across both groups. To our knowledge, this is the first study on DAMAs that examines postdischarge HcRU outside the inpatient setting.

The $0.5 \%$ prevalence of DAMAs in our study was lower than the approximate $1 \%$ to $2 \%$ value that is typically reported in the literature. Prior studies have typically reported results based on mixed-payer populations. ${ }^{3-10}$ These mixed-payer populations include publicly insured (Medicare or Medicaid) or uninsured stays, which account for a disproportionate share of all DAMAs. In contrast, commercially insured stays account for the lowest proportion of all DAMAs. ${ }^{10}$ Similar to prior literature, ${ }^{5}$ the DAMA group in our study was younger, had a higher proportion of males, had a higher comorbidity burden, and had a shorter LOS than the routinely discharged group.

We observed a greater likelihood of ED utilization after a DAMA. Similar findings have been reported, which may indicate that patients with a DAMA receive inadequate treatment at the time of discharge and may require further acute treatment. For example, a prior study reported that, after a DAMA, individuals with asthma were four times more likely to have an ED visit within 14 days compared with those discharged routinely. ${ }^{4}$

Contrary to prior findings, ${ }^{3-9}$ we found no significant difference in the odds of a 30-day inpatient readmission across the DAMA and routine discharge groups, which may be attributable to differences in the populations studied. Those previous studies used mixed payer populations and did not differentiate results by payer type. The mixed payer populations in these studies were older (mean ages, 55 years and above) and had an increased comorbidity burden compared with our commercially insured population. Furthermore, some of these studies were either limited to single sites, ${ }^{8}$ single state hospital systems, ${ }^{3,4,9}$ or focused on specific medical populations. ${ }^{3,4,6-9}$ Our national sample of commercially insured adults is considerably younger, with a mean age of 43 years. Thirty days may be too brief to observe enough inpatient readmissions for the purpose of comparative analyses. This is suggested by our results, which indicated that there is an association between DAMA and 90-day inpatient readmission. Additionally, nonsignificant findings for 30-day inpatient readmissions may also be due to the small sample size of the DAMA group in our study, which may have limited robust statistical inference. Future studies in a larger population of commercially insured individuals with a DAMA are required to confirm these findings.

Nonsignificant differences in the rates of 30-day physician office visits, nonphysician outpatient encounters, and prescription drug fills across both groups may explain the null association with 30-day inpatient readmissions. Prior literature on specific medical populations or individuals with general hospital admissions report that early outpatient follow-up can help prevent 30-day readmissions. ${ }^{20-25}$ In our sample, we observed similar rates of outpatient follow-up across the DAMA and routinely discharged groups. Prior studies based on single hospital sites have reported that, at the time of discharge, a lower proportion of individuals with a DAMA received medication prescriptions and outpatient follow-up plans compared with those discharged routinely. ${ }^{11,12}$ In contrast, we evaluated prescription drug fills and outpatient visits during the postdischarge period, which may explain the difference in findings.

The present study has several strengths. To the best of our knowledge, our study represents the first and largest retrospective analysis of DAMAs in a national sample of commercially insured adults. In addition to a large generalizable sample, we examine HcRU after a DAMA across major points of service over a longitudinal postdischarge period. Our results provide a comprehensive understanding of utilization outcomes in this population including those outside the inpatient setting, which has been the focus of prior literature. These findings can help guide the implementation of appropriate patient- and system-level interventions to optimize DAMA prevention and mitigate the associated utilization burden on the healthcare system in the postdischarge period. ${ }^{26,27}$

Our findings should be interpreted with certain limitations in mind. First, this study used data based on a commercially insured sample of patients and may not be generalizable to pub- 
licly insured or uninsured samples. Second, like prior DAMA studies that used the Nationwide Readmissions Database instead, ${ }^{5-7}$ our study was unable to account for individual-level factors such as race, marital status, family social support, income, health literacy, and activation in self-care. Further, given the limitations of our data, we were unable to control for hospital characteristics such as bed size, urban-rural designation, teaching status, and control (eg, private or government ownership). Despite the use of propensity score methods to balance both comparison groups on observable sources of confounding, we cannot rule out the possibility of residual confounding. Lastly, due to a lack of data on postdischarge mortality outcomes, we could not control for competing risk of death in our analysis. However, in a population with an average age of 43 years, we did not expect high or differential 30- or 90-day postdischarge mortality rates across both groups.

Our findings suggest several important directions for future research. First, it will be useful to examine these associations among publicly insured and uninsured samples in which a DAMA is more prevalent and in which the associations with $\mathrm{HcRU}$ may be more pronounced than they are in the commercially insured population. Secondly, future research should identify subgroups of DAMA patients with an increased propensity for postdischarge HcRU. This can help in the design of individualized outpatient follow-up plans that address patient-specific medical and social needs. Finally, our findings highlight the need for education, practice guidelines, and suitable interventions to help providers in the prevention and management of a DAMA.

\section{References}

1. Alfandre DJ. "I'm going home": discharges against medical advice. Mayo Clin Proc. 2009:84(3):255-260. https://doi.org/10.4065/84.3.255

2. Garland $A$, Ramsey $C D$, Fransoo R, et al. Rates of readmission and death associated with leaving hospital against medical advice: a population-based study. CMAJ. 2013;185(14):1207-1214. https://doi.org/10.1503/cmaj.130029

3. Fiscella K, Meldrum S, Barnett S. Hospital discharge against advice after myocardial infarction: deaths and readmissions. Am J Med. 2007;120(12):10471053. https://doi.org/10.1016/j.amjmed.2007.08.024

4. Baptist AP, Warrier I, Arora R, Ager J, Massanari RM. Hospitalized patients with asthma who leave against medical advice: characteristics, reasons, and outcomes. J Allergy Clin Immunol. 2007;119(4):924-929. https://doi. org/10.1016/j.jaci.2006.11.695

5. Kumar N. Burden of 30-day readmissions associated with discharge against medical advice among inpatients in the United States. Am J Med. 2019;132(6):708-717.e4. https://doi.org/10.1016/j.amjmed.2019.01.023

6. Kwok CS, Walsh MN, Volgman A, et al. Discharge against medical advice after hospitalisation for acute myocardial infarction. Heart. 2019;105(4):315321. https://doi.org/10.1136/heartjnl-2018-313671

7. Patel B, Prousi G, Shah M, et al. Thirty-day readmission rate in acute heart failure patients discharged against medical advice in a matched cohort study. Mayo Clin Proc. 2018;93(10):1397-1403. https://doi.org/10.1016/j. mayocp.2018.04.023

8. Southern WN, Nahvi S, Arnsten JH. Increased risk of mortality and readmission among patients discharged against medical advice. Am J Med. 2012;125(6):594-602. https://doi.org/10.1016/j.amjmed.2011.12.017

9. Onukwugha E, Mullins D, Loh FE, Saunders E, Shaya FT, Weir MR. Readmissions after unauthorized discharges in the cardiovascular setting. Med Care. 2011;49(2):215-224. https://doi.org/10.1097/mlr.0b013e31820192a5

10. Stranges E, Wier L, Merrill CT, Steiner C. Hospitalizations in which Patients Leave the Hospital against Medical Advice (AMA), 2007. HCUP Statistical Brief \#78. Healthcare Cost and Utilization Project, Agency for Healthcare Research and Quality; August 2009. Accessed 04/07 2020.http://www.hcup-us. ahrq.gov/reports/statbriefs/sb78.pdf

\section{CONCLUSION}

Using data from a commercially insured population, we identified associations between a DAMA and postdischarge HcRU. The associations differed by category of HcRU. We identified a positive association with the likelihood of ED utilization but no association with the likelihood of 30-day inpatient readmission or general outpatient utilization. Our results indicate that the examination of inpatient readmissions after a DAMA should not be considered in isolation. The identification of the full range of outpatient and inpatient HcRU after a DAMA in a broad population of patients can improve our understanding of outcomes following a DAMA and support appropriate system-level interventions designed to reduce their prevalence.

\section{Acknowledgments}

The statements, findings, conclusions, views, and opinions contained and expressed in this manuscript are based in part on data obtained under license from IQVIA. Source: IQVIA PharMetrics ${ }^{\circledR}$ Plus January 2006 - December 2015 IQVIA. All Rights Reserved. The statements, findings, conclusions, views, and opinions contained and expressed herein are not necessarily those of IQVIA or any of its affiliated or subsidiary entities.

Disclosures: Dr Onukwugha reports grants from Bayer Healthcare Pharmaceuticals, grants from Pfizer, Inc, and personal fees from Novo Nordisk outside the submitted work. The other authors have nothing to disclose. The views expressed in this article are those of the authors and do not necessarily reflect the position or policy of the US Department of Veterans Affairs, the U.S. Government, or the VA National Center for Ethics in Health Care.

Funding: The authors acknowledge the support of the University of Maryland, Baltimore Institute for Clinical \& Translational Research (ICTR) through the ICTR Voucher Program.

11. Edwards J, Markert R, Bricker D. Discharge against medical advice: how often do we intervene? J Hosp Med. 2013;8(10):574-577. https://doi.org/10.1002/ jhm.2087

12. Stearns $C R$, Bakamjian A, Sattar S, Weintraub MR. Discharges against medical advice at a county hospital: provider perceptions and practice. J Hosp Med. 2017;12(1):11-17. https://doi.org/10.1002/jhm.2672

13. Garland A, Fransoo R, Olafson K, et al. The Epidemiology and Outcomes of Critical Illness in Manitoba. Manitoba Centre for Health Policy; April 2012. Accessed April 7, 2020. http://mchp-appserv.cpe.umanitoba.ca/reference/ MCHP_ICU_Report_WEB_(20120403).pdf

14. Elixhauser A, Steiner C, Harris DR, Coffey RM. Comorbidity measures for use with administrative data. Med Care. 1998;36(1):8-27. https://doi. org/10.1097/00005650-199801000-00004

15. Austin PC. A comparison of 12 algorithms for matching on the propensity score. Stat Med. 2014;33(6):1057-1069. https://doi.org/10.1002/sim.6004

16. Austin PC. Optimal caliper widths for propensity-score matching when estimating differences in means and differences in proportions in observational studies. Pharm Stat. 2011;10(2):150-161. https://doi.org/10.1002/pst.433

17. Austin PC, Mamdani MM. A comparison of propensity score methods: a case-study estimating the effectiveness of post-AMI statin use. Stat Med. 2006;25(12):2084-2106. https://doi.org/10.1002/sim.2328

18. Normand ST, Landrum MB, Guadagnoli E, et al. Validating recommendations for coronary angiography following acute myocardial infarction in the elderly: a matched analysis using propensity scores. J Clin Epidemiol. 2001;54(4):387-398. https://doi.org/10.1016/s0895-4356(00)00321-8

19. Mullahy J. Specification and testing of some modified count data models. J Econometrics. 1986;33(3):341-365. https://doi.org/10.1016/03044076(86)90002-3

20. Halasyamani L, Kripalani S, Coleman E, et al. Transition of care for hospitalized elderly patients_-development of a discharge checklist for hospitalists. J Hosp Med. 2006;1(6):354-360. https://doi.org/10.1002/jhm.129

21. Hernandez AF, Greiner MA, Fonarow GC, et al. Relationship between early physician follow-up and 30-day readmission among Medicare beneficiaries 
hospitalized for heart failure. JAMA. 2010;303(17):1716-1722. https://doi org/10.1001/jama.2010.533

22. Leschke J, Panepinto JA, Nimmer M, Hoffmann RG, Yan K, Brousseau DC Outpatient follow-up and rehospitalizations for sickle cell disease patients. Pediatr Blood Cancer. 2012;58(3):406-409. https://doi.org/10.1002/pbc.23140

23. Misky GJ, Wald HL, Coleman EA. Post-hospitalization transitions: Examining the effects of timing of primary care provider follow-up. J Hosp Med. 2010;5(7):392-397. https://doi.org/10.1002/jhm.666

24. Muus K, Knudson A, Klug MG, Gokun J, Sarrazin M, Kaboli P. Effect of post-discharge follow-up care on re-admissions among US veterans with congestive heart failure: a rural-urban comparison. Rural Remote Health.
2010;10(2):1447.https://doi.org/10.22605/RRH1447

25. Ryan J, Kang S, Dolacky S, Ingrassia J, Ganeshan R. Change in readmissions and follow-up visits as part of a heart failure readmission quality improvement initiative. Am J Med. 2013;126(11):989-994.e1. https://doi.org/10.1016/j.amimed.2013.06.027

26. Alfandre D. Improving quality in against medical advice discharges-more empirical evidence, enhanced professional education, and directed systems changes. J Hosp Med. 2017;12(1):59-60. https://doi.org/10.1002/ jhm. 2678

27. Nagarajan M, Offurum Al, Gulati M, Onukwugha E. Discharges Against Medical Advice: Prevalence, Predictors, and Populations. In: Alfandre D, ed. Against-Medical-Advice Discharges from the Hospital. Springer; 2018:11-29. 